\title{
HARMONIC MAPS DEFINED ON A MANIFOLD WITH A DEGENERATE METRIC
}

\author{
HONG JIAXING
}

(Communicated by Jonathan M. Rosenberg)

\begin{abstract}
Some nonexistence and existence of harmonic maps defined on a manifold with a degenerate metric are proved.
\end{abstract}

\section{INTRODUCTION}

The theory of harmonic maps between Riemannian manifolds has been considered extensively (see, [SE, EL]). Recently the harmonic maps between Lorentzian manifolds have also attracted much attention of many mathematicians (see, $\left.\left[G_{1}, G_{2}, C\right]\right)$. However, for the harmonic maps defined on manifolds with degenerate metrics, very little is known. The partial differential equations describing such harmonic maps defined on manifolds with degenerate metrics are, generally speaking, semilinear and of mixed type. To the author's knowledge, so far there has not been a general method to deal with such kind of partial differential equations. This paper is devoted to the study of harmonic maps defined in a special manifold with a degenerate metric.

Let $R^{2}$ be equipped with a degenerate metric of the form

$$
g=g_{i j} d x^{i} d x^{j}=\left(\left(1-x^{2}\right) \delta^{i j}+x^{i} x^{j}\right) d x^{i} d x^{j} .
$$

Denote this manifold by $\left(R^{2}, g\right)$. Obviously, $g$ is a Riemannian metric inside the unit disk $D$, an indefinite metric outside and degenerate on the unit circle. According to [SE], a harmonic map from $(\Omega, g)$ into $S^{2}$ for some domain $\Omega$ is a critical point of the energy functional of the maps $\varphi: \Omega \rightarrow S^{2}$, i.e.

$$
\int_{\Omega} g^{i j} \sqrt{\left|1-x^{2}\right|} \varphi_{i} \cdot \varphi_{j} d x \quad \text { with } \varphi^{2}=1 .
$$

Its Euler-Lagrangian equation is as follows:

$$
\left(\delta^{i j}-x^{i} x^{j}\right) \varphi_{i j}-2 x^{i} \varphi_{i}+\left(\delta^{i j}-x^{i} x^{j}\right)\left(\varphi_{i} \cdot \varphi_{j}\right) \varphi=0 .
$$

Received by the editors January 4, 1988 and, in revised form, August 29, 1988. 1980 Mathematics Subject Classification (1985 Revision). Primary 53B30.

Key words and phrases. Maximum principle, equation of mixed type, completely integrable system. 
A $C^{2}$ map $\varphi$ is harmonic iff $\varphi$ satisfies (1.3). This is a semilinear system of equations of mixed type. $[\mathrm{H}]$ studied in detail the boundary value problems for Busemann equation, i.e. the linear part of $(1.3)$ and $\left[G_{3}\right]$ also discussed the solvability in the smooth category.

Our main results are as follows:

Theorem 1. Let a given domain $\Omega \subset R^{2}$ contain $\bar{D}$. Then any $C^{2}$ harmonic map from $(\Omega, g)$ into $S^{2}$ is constant.

Theorem 2. For any point $q$ in $D$, there is a $C^{\infty}$ harmonic map from $\left(R^{2} \backslash q, g\right)$ into $S^{2}$, whose range contains at least an interior point.

Further results on boundary value problems of harmonic maps defined on $(\Omega, g)$ for a general domain $\Omega$ of $R^{2}$ will be presented in another paper.

In $\S 2$ using a transformation we reduce the proof of Theorem 1 to that of the nonexistence of nonconstant harmonic maps from a unbounded domain of $R^{2}$ with the Euclidean metric into $S^{2}$. In $\S 3$ based on the transformation introduced in $\S 2$ we shall construct two harmonic maps into $S^{2}$, respectively, defined on some subdomains of the Euclidean plane and Minkowsky plane. Finally, we pull them back to $(D, g)$ and $\left(R^{2} / D, g\right)$ respectively, and smoothly match them on the unit circle. Many techniques we shall use are due to $\left[G_{2}\right]$.

2. NONEXISTENCE OF A HARMONIC MAP FROM $(\bar{D}, g)$ INTO $S^{2}$

Under the polar coordinates $(\theta, \rho)(1.3)$ may be rewritten in the form

$$
\left(1-\rho^{2}\right) \varphi_{\rho \rho}+\frac{1-2 \rho^{2}}{\rho} \varphi_{\rho}+\frac{1}{\rho^{2}} \varphi_{\theta \theta}+\left[\left(1-\rho^{2}\right) \varphi_{\rho}^{2}+\frac{1}{\rho^{2}} \varphi_{\theta}^{2}\right] \varphi=0 .
$$

A change of independent variables

$$
\psi:(\theta, \rho) \rightarrow\left(\theta, \xi=\ln \left[\left(1+\sqrt{1-\rho^{2}}\right) / \rho\right]\right.
$$

transforms $D \backslash\{0\}$ onto $G=\left\{(\theta, \xi) \in R^{2} \mid 0<\xi<+\infty, 0 \leq \theta \leq 2 \pi\right\}$ and meanwhile, reduces $(2.1)$ to

$$
\varphi_{\xi \xi}+\varphi_{\theta \theta}+\left(\varphi_{\xi}^{2}+\varphi_{\theta}^{2}\right) \varphi=0 .
$$

This is nothing else but the equation describing the harmonic maps from the Euclidean plane into $S^{2}$. Note that under the present circumstance, $\varphi$ must be a $2 \pi$-periodic vector function of theta. Introduce a complex variable $z=\theta+i \xi$. From the following relation

$$
\partial_{z}=\frac{1}{2}\left(\partial_{\theta}+i \partial_{\xi}\right), \quad \partial_{\bar{z}}=\frac{1}{2}\left(\partial_{\theta}-i \partial_{\xi}\right) .
$$

(2.3) may be put in the form

$$
\varphi_{z \bar{z}}+\left(\varphi_{z} \cdot \varphi_{\bar{z}}\right) \varphi=0
$$


Multiplying both sides of $\left(2.3^{\prime}\right)$ by $\varphi_{z}$ and using the property: $\varphi^{2}=1$ we can find

$$
\left(\varphi_{z}^{2}\right)_{\bar{z}}=0 \text { or } \varphi_{z}^{2}=f(z) .
$$

$\left(2.4^{\prime}\right)$ was first obtained in $\left[G_{2}\right]$. This shows that $4 \varphi_{z}^{2}=u+i v$ is an analytic function and

$$
\varphi_{\theta}^{2}-\varphi_{\xi}^{2}=u, \quad 2 \varphi_{\theta} \cdot \varphi_{\xi}=v .
$$

We claim that $u$ and $v$ are constants if $\varphi$ is a $C^{2}$ harmonic map from $(\bar{D}, g)$ into $S^{2}$. Indeed we may view $u$ and $v$ as two harmonic functions defined in $R_{+}^{2}$ since $\varphi(\theta, \xi)$ is periodic in $\theta$. From (2.2) it is easy to see

$$
\begin{aligned}
& v(\theta, 0)=0 \text { and } u(\theta, \xi), v(\theta, \xi) \text { uniformly } \\
& \text { with respect } \theta \text { approach to zero if } \xi \rightarrow+\infty .
\end{aligned}
$$

By means of the symmetric principle we may extend $v$ as a harmonic odd function of $\xi, \tilde{v}$, defined on the whole plane. Therefor $v=\tilde{v}=$ constant in $\bar{R}_{+}^{2}$ since $\tilde{v}$ is bounded in $R^{2}$. This implies that $u$ is constant in $\bar{R}_{+}^{2}$ too. (2.6) and (2.5) show that these constants are zero. So far we have proved the previous assertion. Indeed we have

Lemma 2.1. Let $\varphi$ be a $C^{2}$ harmonic map from $(\Omega=\bar{D} \backslash\{0\}, g)$ into $S^{2}$ and $\sup \rho|\nabla \varphi|<+\infty$. Then $\varphi_{z}^{2}=$ constant on $G$, i.e., $\varphi$ is a normalized harmonic map defined on $G$.

According to $\left[G_{2}\right]$, a harmonic map $\varphi$ such that $\varphi_{z}^{2}=$ constant is called a normalized harmonic map. We shall discuss two cases of (2.5). Set

$$
\left.\Sigma=\{\theta, \xi) \in R_{+}^{2} \mid \varphi_{\xi}=0\right\} .
$$

We shall list a result in $\left[G_{2}\right]$ without proof.

Lemma 2.2. Let $u=1$ and $v=0$ in (2.5). Then in $R_{+}^{2} \backslash \Sigma$ with $\operatorname{sh}(\lambda / 2)=$ $\sqrt{\varphi_{\xi}^{2}}, m=\varphi_{\xi} /\left|\varphi_{\xi}\right|, n=\varphi_{\theta} /\left|\varphi_{\theta}\right|$ and $W=\left(\varphi^{\mathrm{t}}, m^{\mathrm{t}}, n^{\mathrm{t}}\right)^{\mathrm{t}}$ we have

$$
W_{\xi}=A W \quad \text { where } A=\left(\begin{array}{ccc}
0 & \operatorname{sh} \frac{\lambda}{2} & 0 \\
-\operatorname{sh} \frac{\lambda}{2} & 0 & -\frac{1}{2} \lambda_{\theta} \\
0 & \frac{1}{2} \lambda_{\theta} & 0
\end{array}\right)
$$

and

$$
W_{\theta}=B W \quad \text { where } B=\left(\begin{array}{ccc}
0 & 0 & \operatorname{ch} \frac{\lambda}{2} \\
0 & 0 & \frac{1}{2} \lambda_{\xi} \\
-\operatorname{ch} \frac{\lambda}{2} & -\frac{1}{2} \lambda_{\xi} & 0
\end{array}\right) \text {. }
$$

Here $\lambda$ satisfies

$$
\Delta \lambda=-\operatorname{sh} \lambda, \quad \text { in } R_{+}^{2} \backslash \Sigma
$$


For the second case we have

Lemma 2.3. Let $u=v=0$ in (2.5). Then in $R_{+}^{2} \backslash \Sigma$ with $\lambda=\sqrt{\varphi_{\xi}^{2}}, m, n$ and $W$ as defined in Lemma 2.2 we have

$$
W_{\xi}=A W \quad \text { where } A=\left(\begin{array}{ccc}
0 & \lambda & 0 \\
-\lambda & 0 & -\frac{\lambda_{\theta}}{\lambda} \\
0 & \frac{\lambda_{\theta}}{\lambda} & 0
\end{array}\right)
$$

and

$$
W_{\theta}=B W \quad \text { where } B=\left(\begin{array}{ccc}
0 & 0 & \lambda \\
0 & 0 & \frac{\lambda_{\xi}}{\lambda} \\
-\lambda & -\frac{\lambda_{\xi}}{\lambda} & 0
\end{array}\right)
$$

Here $\lambda$ satisfies

$$
\Delta \lambda^{2}=4|\nabla \lambda|^{2}-2 \lambda^{4} \quad \text { in } R_{+}^{2} \mid \Sigma .
$$

Proof. On the open set $R_{+}^{2} \backslash \Sigma, \varphi, m, n$ are $C^{\infty}$-continuous. Directly differentiating $m, n$ with respect to $\xi, \theta$ we find

$$
W_{\xi}=A W \quad \text { where } A=\left(\begin{array}{ccc}
0 & \lambda & 0 \\
-\lambda & 0 & -\sigma \\
0 & \sigma & 0
\end{array}\right) \text { with } \sigma=m n_{\xi} .
$$

and

$$
W_{\theta}=B W \quad \text { where } B=\left(\begin{array}{ccc}
0 & 0 & \lambda \\
0 & 0 & \mu \\
-\lambda & -\mu & 0
\end{array}\right) \text { with } \mu=n \cdot m_{\theta} .
$$

From $W_{\xi \theta}=W_{\theta \xi}$ it follows that

$$
A_{\theta}-B_{\xi}+A B-B A=0 \text { in } R_{+}^{2} \backslash \Sigma .
$$

This implies (2.10), (2.11) and (2.12) at once. Lemma 2.3 is proved.

Now we are in a position to complete the proof of Theorem 1 .

The end of the proof of Theorem 1. First of all, from (2.2) and the fact that $\varphi$ is in $C^{2}(\bar{D})$ it follows that

$$
\lambda^{2}=\left|\varphi_{\xi}^{2}\right| \leq C \exp (-2 \xi) \quad \text { in } \bar{R}_{+}^{2}
$$

for some constant $C$. We claim:

There is a sufficiently large $k$ such that

$$
e=\lambda^{2} /(1+\xi)^{k}=0 \text { in } \bar{R}_{+}^{2} .
$$

If (2.17) is true, then the assertion of Theorem 1 comes immediately from (2.17) and (2.5). Let us now verify (2.17). Suppose that $e$ attains its maximum at $\left(\theta^{*}, \xi^{*}\right)$ where $\xi^{*}$ in $(0,+\infty)$. This is possible from $(2.16)$. If $\lambda\left(\theta^{*}, \xi^{*}\right)=0$, (2.17) is proved. So we shall only discuss the case $\lambda\left(\theta^{*}, \xi^{*}\right) \neq 0$. In fact we have at $\left(\theta^{*}, \xi^{*}\right)$

$$
\begin{gathered}
2(1+\xi)^{-k} \lambda \lambda_{\xi}-k(1+\xi)^{-k-1} \lambda^{2}=0 \\
2(1+\xi)^{-k} \lambda \lambda_{\theta}=0
\end{gathered}
$$


and

(2.20) $0 \geq \Delta e=(1+\xi)^{-k}\left[4 \lambda_{\xi}^{2}-2 \lambda^{4}-4 k(1+\xi)^{-1} \lambda \lambda_{\xi}+k(k+1)(1+\xi)^{-2} \lambda^{2}\right]$.

In getting (2.20) we have used (2.12). Combining (2.18)-(2.20) we find at $\left(\theta^{*}, \xi^{*}\right)$

$$
\lambda^{2}\left(2 \lambda^{2}-k(1+\xi)^{-2}\right) \geq 0
$$

Take $k$ so big that

$$
k \geq \sup _{\bar{R}_{+}^{2}}\left(2 \lambda^{2}(1+\xi)^{2}+1\right) .
$$

(2.16) guarantees that the right-hand side of the last inequality is finite. Now $\lambda\left(\theta^{*}, \xi^{*}\right)=0$ follows at once from (2.21). So (2.17) is proved. This completes the proof of Theorem 1 .

\section{EXISTENCE OF HARMONIC MAPS}

This section is concerned with the construction of the harmonic map mentioned in Theorem 2. From a slight computation we can see that the rotation and the hyperbolic transformation, i.e., for any $q=\left(q^{1}, q^{2}\right)^{t} \in D$

$$
\bar{x}=\frac{\left(1-q^{2}\right)^{1 / 2}}{1-q^{t} \cdot x}\left(\delta^{i j}-q^{i} q^{j}\right)^{-1 / 2}(x-q)
$$

keep the unit circle and are conformal with respect to $g$ outside the unit circle. So we may assume $q=0$, if necessary, making a change of variables as mentioned in (3.1). Before describing the construction of the harmonic map we expected, the connection between harmonic maps from $(\bar{D} \backslash\{0\}, g)$ and from $\bar{G}$ into $S^{2}$ must be studied.

Lemma 3.1. Let $\varphi$ be a smooth harmonic map from $\bar{G}$ into $S^{2}$ and let $\varphi$ be $2 \pi$-periodic in $\theta$, and $\varphi_{\xi}(\theta, 0)=0$ for all $\theta$ in $[0,2 \pi]$. Then $\varphi \circ \psi$ is $a$ smooth harmonic map from $(\bar{D} \backslash\{0\}, g)$ into $S^{2}$.

Proof. From the hypothesis in the present lemma it is evident that $\varphi \circ \psi$ is a smooth harmonic map from $(D \backslash\{0\}, g)$ into $S^{2}$. So we shall only prove that $\varphi \circ \psi$ is smooth up to the unit circle. First of all there is no difficulty in proving $\partial_{\theta}^{k} \varphi \in C(\bar{D} \backslash\{0\})$ for each $k \in z^{+}$. Furthermore,

$$
\begin{aligned}
\varphi_{\rho} & =-\varphi_{\xi} / \rho \sqrt{1-\rho^{2}} \\
& =-\varphi_{\xi} \operatorname{ch}^{2} \xi / \operatorname{sh} \xi \rightarrow-\varphi_{\xi \xi}(\theta, 0) \text { if } \rho \rightarrow 1 .
\end{aligned}
$$

Meanwhile

$$
\partial_{\theta}^{k} \varphi_{\rho} \rightarrow-\partial_{\theta}^{k} \varphi_{\xi \xi}(\theta, 0) \quad \text { if } \rho \rightarrow 1
$$


Now we proceed to discuss the continuity of $\varphi_{\rho \rho}$ and $\partial_{\theta}^{k} \varphi_{\rho \rho}$. From (3.2) and integrating $(2.1)$ we find

$$
\begin{aligned}
\varphi_{\rho} & =\frac{-1}{\rho \sqrt{1-\rho^{2}}} \int_{1}^{\rho} \sqrt{1-\bar{\rho}^{2}} \bar{\rho} \varphi_{\bar{\rho}}^{2} \varphi d \bar{\rho}+\frac{1}{\rho \sqrt{1-\rho^{2}}} \int_{1}^{\rho} \frac{f(\theta, \bar{\rho})}{\sqrt{1-\bar{\rho}^{2}}} d \bar{\rho} \\
& =I_{1}+I_{2}
\end{aligned}
$$

where

$$
f=-\frac{1}{\rho}\left(\varphi_{\theta \theta}+\varphi_{\theta}^{2} \varphi\right)
$$

(3.3) guarantees $\partial_{\theta}^{k} \partial_{\rho} f \in C(\bar{D} \backslash\{0\})$. Let us first consider $I_{2}$. A change of the variable of integration: $\bar{\rho} \rightarrow 1-\lambda(1-\rho)$ yields

$$
I_{2}=\frac{1}{\rho \sqrt{1+\rho}} \int_{1}^{0} \frac{f(\theta, 1-\lambda(1-\rho))}{\sqrt{\lambda} \sqrt{2-\lambda(1-\rho)}} d \lambda .
$$

Thus $\partial_{\rho} \partial_{\theta}^{k} I_{2} \in C(\bar{D} \backslash\{0\})$. Analogously, an application of the previous change of the variables of integration gives

$$
I_{1}=\int_{0}^{1} g(\rho, \lambda)(1-\rho) \varphi_{\rho}^{2} \varphi(\theta, 1-\lambda(1-\rho)) d \lambda
$$

where $g(\rho, \lambda)=-(1-\lambda(1-\rho)) \sqrt{2-\lambda(1-\rho)} / \rho \sqrt{1+\rho}$ is smooth near $\rho=1$. Let us evaluate $\Delta_{h} \varphi_{\rho}=\left(\varphi_{\rho}(\theta, \rho-h)-\varphi_{\rho}(\theta, \rho)\right)$ for all $\rho \leq 1$ and $h \geq 0$. From (3.4) we have

$\Delta_{h} \varphi_{\rho} \leq \int_{0}^{1}\left|\tau_{h}((1-\rho) g \varphi)\right| \tilde{\Delta}_{h}\left(\varphi_{\rho}^{2}\right)(\rho, h) d \lambda+\int_{0}^{1}\left|\varphi_{\rho}^{2}\right|\left|\Delta_{h}((1-\rho) g \varphi)\right| d \lambda+\left|\Delta_{h} I_{2}\right|$ where $\tau_{h} u(\rho, h)=u(\theta, \rho-h)$ and $\tilde{\Delta}_{h}(f)(\rho, h)=\max \left\{\left|\Delta_{\bar{h}} f(\theta, \bar{\rho})\right|, \rho \leq \bar{\rho} \leq 1\right.$ and $0 \leq \theta \leq 2 \pi, 0 \leq \bar{h} \leq h\}$. Hence

$$
\left|\Delta_{h} \varphi_{\rho}\right| \leq C_{1}(1-\rho+h) \tilde{\Delta}_{h}\left(\varphi_{\rho}\right)(\rho, h)+C_{1} h .
$$

Thus

$$
\tilde{\Delta}_{h}\left(\varphi_{\rho}\right)(\rho, h) \leq 2 C_{1} h \text { if } h \text { and }(1-\rho) \text { are all less than } 1 / 4 C_{1}
$$

which implies

$$
\left|\Delta_{h} \varphi_{\rho} / h\right| \leq 2 C_{1} \text { if } h \text { and }(1-\rho) \text { all }<1 / 4 C_{1} .
$$

This means $\varphi_{\rho}$ is Lipschitz continuous if $\rho \leq 1$. So we can, under the sign of integration, differentiate (3.4) with respect to $\rho$. As a result

$$
\lim _{\rho \rightarrow 1} \varphi_{\rho \rho}=-g(1,0) \varphi_{\rho}^{2} \varphi(\theta, 1)+\left.\partial_{\rho} I_{2}\right|_{\rho=1} .
$$

By a similar argument we can prove the continuity of $\partial_{\theta}^{k} \varphi_{\rho \rho}$ and $\partial_{\theta}^{k} \partial_{\rho}^{j} \varphi_{\rho \rho}$ near 1 for all $j, k \geq 0$. This completes the proof of Lemma 3.1. 
Remark 3.2. The unit circle is the characteristic for (2.1). But from (2.1) $\varphi_{\rho}$ on the unit circle is uniquely decided by the value $\varphi(\theta, 1)$, if $\varphi$ is in $C^{1}(\bar{D})$. From (3.8) one can see that $\varphi_{\rho \rho}$ is uniquely decided by the value on $\partial D$ of $\varphi, \varphi_{\rho}$ and $\varphi_{\rho \theta}, \varphi_{\rho \theta \theta}$. So it is done by $\varphi(\theta, 1)$ if all the derivatives involved are continuous in $\bar{D}$. By a similar argument one can prove that $\partial_{\rho}^{k} \varphi$ for all $k>2$ are completely decided by $\varphi(\theta, 1)$ if $\partial_{\theta}^{k} \varphi(\theta, \rho)$ are continuous in $\bar{D}$ for all $k$.

Remark 3.3 The transform

$$
\psi_{1}:(\theta, \rho) \rightarrow\left(\theta, \xi=\left(\cos ^{-1}(1 / \rho)\right)\right)
$$

maps $R^{2} \backslash D$ onto $G_{1}=\{0 \leq \theta \leq 2 \pi, 0 \leq \xi<\pi / 2\}$ and the unit circle onto the segment $\{\xi=0,0 \leq \theta \leq 2 \pi\}$. Under the new coordinates (2.1) may be rewritten in the form

$$
\varphi_{\theta \theta}-\varphi_{\xi \xi}+\left(\varphi_{\theta}^{2}-\varphi_{\xi}^{2}\right) \varphi=0 \text { in } G_{1} .
$$

Let $\varphi$ be a solution smooth in $G_{1}$ and $2 \pi$-periodic in $\theta$ and let $\varphi_{\xi}(\theta, 0)=0$. Then $\varphi \circ \psi_{1}$ is a smooth harmonic map from $\left(R^{2} \backslash D, g\right)$ into $S^{2}$.

Now we proceed to construct the harmonic map from $(G, E)$ (or $(G, L)$ ) into $S^{2}$. Here $E$ and $L$ stand, respectively, for Euclidean metric and Lorentzian metric. It is evident that (2.7)-(2.9) form a completely integrable system. But the difficulty in constructing a harmonic map from $(G, E)$ into $S^{2}$ is that $\varphi$ must be $2 \pi$-periodic in $\theta$. Next we shall explain in detail this procedure. Many techniques later used are due to $\left[G_{2}\right]$.

The first step is to find a function $\lambda=\lambda(\xi)$ which is a solution of $(2.9)$

$$
\lambda^{\prime \prime}+\operatorname{sh} \lambda=0 \text {. }
$$

Multiplying the last equation by $\lambda^{\prime}$ we have

$$
\left(\left(\lambda^{\prime}\right)^{2} / 2\right)^{\prime}+(\operatorname{Ch} \lambda)^{\prime}=0 .
$$

An integration of it yields a first integral

$$
\left(\lambda^{\prime}\right)^{2}+2 \operatorname{ch} \lambda=C_{0}
$$

where $C_{0}$ is a constant to be determined but bigger than 2 . Directly integrating (3.11) we can obtain a smooth periodic solution in $[0,+\infty) \lambda=\lambda(\xi)$ with $\lambda(0)=0$.

The second step is to find a $2 \pi$-periodic solution in $\theta$ of (2.8) for any $\xi$ in $[0,+\infty)$. This is possible. In fact the matrix $B$ in $(2.8)$ has eigenvalues

$$
\mu_{1}=0, \quad \mu_{2}=i \sqrt{C_{0}+2} / 2, \quad \mu_{3}=-i \sqrt{C_{0}+2} / 2 .
$$

In getting (3.12) we have used (3.11) and the formula $\operatorname{ch} \lambda=2 \operatorname{ch}^{2}(\lambda / 2)-1$. Fix now the constant $C_{0}=4 k^{2}-2$ for any integer $k \geq 2$. Thus $\mu_{2}=i k$ and 
$\mu_{3}=-i k$. Meanwhile there is a smooth, real orthogonal matrix

$$
T=\left(\begin{array}{ccc}
\frac{\lambda^{\prime}}{2 k} & \frac{1}{k} \operatorname{ch} \frac{\lambda}{2} & 0 \\
-\frac{1}{k} \operatorname{ch} \frac{\lambda}{2} & \frac{\lambda^{\prime}}{2 k} & 0 \\
0 & 0 & 1
\end{array}\right)
$$

such that

$$
T^{t} B T=\left(\begin{array}{ccc}
0 & 0 & 0 \\
0 & 0 & k \\
0 & -k & 0
\end{array}\right) .
$$

There is no difficulty in checking

$$
T^{\prime}-A T=0 .
$$

Inserting (3.14) into (2.8) we can get a solution of the form

$$
W=T\left(\begin{array}{ccc}
1 & 0 & 0 \\
0 & \cos k \theta & \sin k \theta \\
0 & -\sin k \theta & \cos k \theta
\end{array}\right) a
$$

for any constant vector $a=\left(a_{1}, a_{2}, a_{3}\right)^{\mathrm{t}}$. An application of (3.15) to $W$ in (3.16) provides at once that $W$ satisfies (2.7) for each $a$. Setting, for any unit orthogonal frame $\left(\varphi_{0}, m_{0}, n_{0}\right)$,

$$
\varphi=(2 k)^{-1} \lambda^{\prime} \varphi_{0}+k^{-1} \cos k \theta \operatorname{ch}(\lambda / 2) m_{0}+k^{-1} \sin k \theta \operatorname{ch}(\lambda / 2) n_{0}
$$

we shall check that $\varphi$ is just the harmonic map from $\left(\bar{R}_{+}^{2}, E\right)$ into $S^{2}$ satisfying the assumptions in Lemma 3.1. A direct computation gives $\varphi_{\xi}^{2}=\operatorname{sh}^{2}(\lambda / 2)$ and $\varphi_{\theta}^{2}=\operatorname{ch}^{2}(\lambda / 2)$. By the construction of $\lambda$ it is easy to see $\varphi_{\xi}(\theta, 0)=0$ and $\Sigma=\left\{(\theta, \xi) \in R_{+}^{2} \mid \varphi_{\xi}=0\right\}$ has no interior point. Obviously, $R_{+}^{2} \backslash \Sigma$ is an open set and $\varphi, \varphi_{\theta}, \varphi_{\xi}$ are linearly independent on it. In the other hand, $\varphi_{z}\left(\varphi_{z \bar{z}}+\left(\varphi_{z} \cdot \varphi_{\bar{z}}\right) \varphi\right)=0 \varphi_{\bar{z}}\left(\varphi_{z \bar{z}}+\left(\varphi_{z} \cdot \varphi_{\bar{z}}\right) \varphi\right)=0$ since $\varphi^{2}=1$. This implies that $\varphi$ satisfies $(2.3)$ on $R_{+}^{2} \backslash \Sigma$. So does it in $R_{+}^{2}$ since $\varphi$ is smooth in $\bar{R}_{+}^{2}$ and $\Sigma$ has no interior point. Now an application of Lemma 3.1 to $\varphi$ yields that $\varphi \circ \psi$ is a smooth harmonic map from $(\bar{D} \backslash\{0\}, g)$ into $S^{2}$.

The end of the proof of Theorem 2. Set $u_{0}(\theta)=\varphi(\theta, 0)$ and $u_{1}(\theta)=0$ where $\varphi$ is the map just now constructed and defined on $\bar{R}_{+}^{2}$. Evidently, $u_{0}(\theta)$ is smooth and $2 \pi$-periodic, and $\dot{u}_{0} \cdot u_{0}=0$. Consider a Cauchy problem (3.10) with

$$
\varphi(\theta, 0)=u_{0}(\theta) \text { and } \varphi_{\xi}(\theta, 0)=0 .
$$

According to the result in $\left[G_{1}\right]$ this Cauchy problem admits a solution $\varphi$ in $C^{\infty}\left(R^{1} \times[0, \pi / 2]\right)$. From Remark 3.3 it follows that $\varphi \circ \psi_{1}$ is a harmonic map from $\left(R^{2} \backslash D, g\right)$ into $S^{2}$. By the construction we know $\varphi \circ \psi$ and $\varphi \circ \psi_{1}$ continuously match on the unit circle. Remark 3.2 shows that they smoothly match on the unit circle. Theorem 2 is proved. 


\section{ACKNOWLEDGMENT}

The author would like to thank Professor Abdus Salam, and International Atomic Energy Agency UNESCO for hospitality at the International Centre for Theoretical Physics, Trieste. The author is also grateful to Professor James Eells for his encouragement.

\section{REFERENCES}

[SE] J. H. Sampson and J. Eells, Harmonic mappings of Riemannian manifolds, Amer. J. Math. 86 (1964), 109-160.

[EL] J. Eells and L. Lemaire, A report on harmonic maps, Bull. London Math. Soc. 10 (1978), 1-68.

$\left[\mathrm{G}_{1}\right]$ ] C. $\mathrm{H} . \mathrm{Gu}$, On the Cauchy problem for harmonic maps defined on two dimensional Minkowsky space, Comm Pure Appl. Math. 33 (1980), 727-737.

$\left[\mathrm{G}_{2}\right]$ - On the harmonic map from $R^{1.1}$ to $S^{1.1}$, Proceedings of the 1982 Changchun symposium on differential geometry and differential equations, Science Press, Beijing, China, 1986.

$\left[\mathrm{G}_{3}\right]$ _. On partial differential equations of mixed type in $n$ independent variables, Comm. Pure Appl. Math. 34 (1981), 333-345.

[C] Y. Choquet Bruhat, Global existence theorems for hyperbolic harmonic maps, Ann. Inst. H. Poincaré 46 (1987), 97-111.

[H] L. K. Hua, A talk starting from the unit circle, Science Publisher Beijing, China, 1977.

Institute of Mathematics, Fudan University, Shanghai, China 